\title{
Dynamic Capability, Ambidexterity, Social Network--Empirical Evidence from SMEs in China
}

\author{
Xin $\mathrm{He}^{1}$ \\ School of Management, \\ Lanzhou University \\ Hexin85@1zu.edu.cn
}

\author{
$\mathrm{Xia} \mathrm{Wu}$ \\ School of Management, \\ Lanzhou University \\ 1209132335@qq.com
}

\author{
Yanhai Zhao ${ }^{1}$ \\ School of Management, \\ Lanzhou University \\ yhzhao@lzu.edu.cn
}

\author{
Dave Croasdell ${ }^{2}$ \\ University of Nevada, \\ Reno \\ davec@unr.edu
}

\begin{abstract}
The investigation of organization's ambidexterity is a challenge in the researches of management sciences. As existent literature showed a positive relation between dynamic capability and innovation, few empirical studies are conducted to explain how dynamic capability impacts on the balanced and combined dimension of ambidexterity, and still less on how social network moderates this relation. By a relational model of dynamic capability, ambidexterity, and social network, this study has conducted multiple regression analysis on the data collected from 350 SMEs in mainland China. The results show that, dynamic capability has positive influence on both the combined and balanced dimension of ambidexterity; and both the relational network and structural network play an inverted $U$ moderating role, where the moderation of relational network is stronger than that of structural network. This study provides empirical support on dynamic capability's influence on ambidexterity together with the moderation of social network.
\end{abstract}

\section{Introduction}

In the turbulent business environment nowadays, firms must engage in both exploratory and exploitative behavior to establish sustained competitive advantage, that is, organization's ambidexterity (AM). Danneels argues that there is both exploratory and exploitative innovation in the field of technological innovation [1]. Meanwhile, these two types of innovation are of distinctive nature, where exploratory innovation (EPI) seeks for completely new measures from current technology and practice, exploitative innovation (ETI) realizes gradual improvement based on current technology and practice. As a result, how to coordinate these two forms of innovation is becoming a research focus. Based on the approach of dynamic capability (DC), Teece considers that DC can not only allocate the resources in a valued manner to realize product innovation, but also can help firms to adapt to the changing competitive environment through flexible internal process by way of integrating and restructuring of internal and external resources [2]. However, the existent empirical studies have focused on DC's respective influence either on exploratory and exploitative innovation [3], but very few on the joint influence of the two. Hence, it is necessary to deepen the investigation of DC's influence on the balanced dimension (BA) and the combined dimension (CA) of ambidextrous innovation.

As innovation is a collective and societal behavior, firms need social network to acquire necessary resources, knowledge, ideas and information for innovation. In the business and research context of China, social network (SN) is often regarded as an informal "guanxi (relation)" based on trust and reputation, where this "guanxi" is indispensable in innovation and technological development. So far, researchers have not reached a consensus regarding SN' influence on innovation. On one hand, the establishment of SN is an important strategy to acquire key technological knowledge to promote innovation [4]. On the other hand, social network can also limit the innovation of its members, thus constraining innovative activities. And because of this, the influence of $\mathrm{SN}$ on innovation is not effective in a linear manner, where when social network, at a medium level, will have the most positive influence on innovation, and but while the intensity and concentration of social network is intensified, the relative cost (the time, energy and other resources necessary for developing and maintaining the $\mathrm{SN}$ ) will also increase, leading to the exhaustion of payoffs gained by increased cost, and thus hindering further innovative activities [5]. Taking into account the important influence of $\mathrm{SN}$ on 
innovation, this study supposes that social network can also play a significant moderating role in the DCAmbidexterity relation. Thus, the second objective of this study is to investigate how SN can moderate the DC-Ambidexterity relation with empirical evidence.

Firms, small or big, are all faced with problems originated from the AM. However, most of ambidexterity studies have focused on larger corporations [6]. Existent studies have shown that, as small and medium enterprises (SME) are characteristically different from these big firms in terms of resource, management experiences and R\&D input, they will have different strategic approaches, where SMEs are inclined to improve their performance by the balance of exploratory and exploitative innovation, while the larger corporations will lay emphasis on the combination of the two [7]. As the two types of firms are distinctive in terms of ambidexterity, this study focuses on SMEs to understand how they SMEs use their DC, together with social network, to realize the balanced development and mutual promotion of both exploratory and exploitative innovation.

In summary, based on the existent contributions, and by building a moderated theoretical model this study aims to explore the functional mechanism of the influence of SMEs' DC on the balanced and combined dimension of ambidextrous innovation, and also the moderation of social network on this. This is for the purpose of enriching and supporting the theories of DC and ambidexterity, and to provide guidance in how SME can use DC to break through the dilemma of ambidexterity. And as the samples are all from the SMEs of mainland China, it is expected that this research can be helpful for SMEs in the emerging economies.

\section{Theoretical Foundation and Research Hypotheses}

\subsection{Ambidexterity, dynamic capability and the main effect of the two}

Researches have proven that exploration and exploitation are two types of innovation of quite distinct nature [8], where they need specific context, organizational structure and background. The exploratory innovation (EPI) is for the purpose to meet the needs of new customers' and markets by way of breaking away from current technology to innovate new product or service. For those firms engaging in exploratory innovation, they are more flexible to adapt to the changing environment for success. The exploitative innovation (ETI), for its part, aims to meet current customer and market needs by way of utilizing and perfecting current available knowledge and techniques, improving current product or service, which will make the current production process more effective [9]. Some studies support this with the argument that, firms should simultaneously engage in both EPI and ETI to establish sustained competitive advantage.

Cao has provided a categorization of balanced dimension and combined dimension of ambidexterity, where the BA signifies the relative balance of the two, while the CA means that the EPI and ETI should mutually supplement and promote each other so as to make up deficiencies of each other and to amplify the value created by each one [10]. The ideal status will then be that the protocols developed through ETI can be integrated into those necessary for EPI, and thus provide a resource base for ETI. In the same manner, high-level of ETI can effectively improve the new products developed through EPI, which will help its commercialization. However, because the two innovations have, by nature, different requirements in structure, process, strategy and ability, and this is rather challenging for SMEs, which are lack of resources and operational experiences to engage in both of these two innovations [11]. As a result, it will be conditional that SME could realize both $\mathrm{CA}$ and $\mathrm{BA}$ of ambidextrous innovations.

DC can be defined as firm's capability to integrate, construct and reallocate resources, assets and abilities to respond to (or bring about) market changes [2,12]. It is also considered to realize sustained innovation and change through integration and reallocation of resources [12]. The existent studies have shown that, DC has positive influences on both EPI and ETI [13]. In his review discussing the antecedents of ambidexterity, Asif classified DC as one of the antecedents of AM, arguing that DC can not only trigger, but also orchestrate AM [14]. In a case study, Carrick also demonstrated how life sciences firms can use DC to develop R\&D resources [15].

In the extension of above studies and evidences, we think that DC has significant positive influence on SME's EPI and ETI, and it can also have positive influence on the $\mathrm{CA}$ and BA. Following are our research hypotheses:

Hla: there is a positive relation between DC and the BA (balanced dimension) of AM (ambidextrous innovation).

$H 1 b$ : there is a positive relation between $D C$ and the $C A$ (combined dimension) of $A M$. 


\subsection{Social network and its influence on the main effect}

\section{Social network}

As an informal social structure [16], social network (SN) refers to the informal relation network between firms and their business partners, friends and relatives so as to make up the insufficiency of formal business interactions in promoting business exchanges. The members of the network can be government departments, supervision institutions, clients, suppliers, sales agents, mother companies, higher education institutions, and research institutions [17]. Their cooperation can be voluntary, non-contractual and very close. Social network can be divided into relational and structural types [18]. Relational network (RN) emphasizes the relation quality among the network members, reflecting the high-quality cohesive informal social interaction of the network members of a certain organization. While the structural network (STN) places emphasis on the overall structure of the network, reflecting mainly the positional influence of the network members.

\section{Relational network's influence on main effect}

$\mathrm{RN}$ can often be measured in terms of relational intensity, i.e. the intensity of relation closeness of SN members [19]. In our study, the moderation of $\mathrm{RN}$ on the DC-AM relation can be both positive and negative.

From the positive side, closer RN can be helpful for SMEs' DC to promote AM. The reasons are as the following: 1) an atmosphere of trust can enhance a firm's ability to sense and size the opportunities in the environment, and can also reallocate its resource basis in a more effective manner [20]. Trust can also significantly upgrade the diffusion and flow of explicit knowledge as well as the more complicated implicit knowledge so that firms can continuously acquire the abilities to obtain, integrate and reallocate resources, and the speed of resource transformation can also be accelerated with the mutual trust and frequent interaction among the network members. 2) Close network can control, at a certain level, the behavior of the network members, which will reduce the threats of opportunism [21]. To avoid risks, SMEs are more inclined to engage in ETI, and this is because the EPI is often characterized as with high risk and market uncertainty, where decision-making errors will lead to immediate failure or survival crisis. However, if the network members establish solid relationships, this can reduce the risks of opportunism-related risks. From this, SMEs can relatively control their coordination relationship with each other, and thus the risks and costs together. From our prospective, under the same level promotion of $\mathrm{DC}$, high-quality $\mathrm{RN}$ can reduce the risks of EPI, and thus realize the balanced development of both EPI and ETI in SMEs. And at the same time, the upgrading of the EPI can provide a larger vision for ETI, and thus in turn helps to realize the combined dimension of AM.

From the negative side, closer $\mathrm{RN}$ can also reduce the positive effect of $\mathrm{DC}$ on the $\mathrm{CA}$ and $\mathrm{BA}$ of ambidexterity. The reasons are as the following: 1) the information and resources obtained from too frequent interactions can be repetitive and redundant [22]. For some scholars, the combined effectiveness of resources has its upper limit. Then if the firms integrated and reallocate those repetitive and redundant information, the values created on innovation can thus be very limited and even disappear. And except those extra costs caused by identifying and sorting out redundancy, this also occupies the resources for ETI, which will make it harder to realize the BA and CA. 2) the establishment and maintenance of relations with the network members creates also costs [23]. With the time going, it will consume more of SMEs' time and energy to amend and enforce this interaction mode, thus exhausting the firm's time and energy to integrate or realize more innovative ideas, which will eventually constrain innovative activities in this firm. 3) a too close relation will result in rigidity of the network [24]. Often, the more solid relationship of the network member is, the stronger the conformist mentality will be. And this will limit the members to seek heterogeneous resources necessary for innovation, and also will limit such innovative activities as environment scanning, problem-identifying and innovative problem-solving [5].

From the synthesis of the above two contradictory influences, we suppose that, if the closeness of $\mathrm{RN}$ is under the critical value, it will reinforce DC's promotion in SMEs of BA and CA of ambidexterity; and with the closeness of $\mathrm{RN}$ reaches and surpasses the critical value, the moderate effect of $\mathrm{RN}$ will be reverted to reduce such promotion. The research hypotheses are as the following:

$H 2 a$ : $R N$ plays an inverted $U$ moderation role in the $D C-B A$ relation.

$H 2 b: R N$ plays an inverted $U$ moderation role in the $D C-C A$ relation.

\section{Structural network's influence on main effect}

It is suggested by scholars that STN is for the purpose of observing the location and distribution of information flows in the network [25], and while a firm is in an ideal position, it can obtain more diversified and richer information. In this study, the meaning of the STN is mainly its "location", which include, the distance and speed that this firm can obtain resources and the influence of this firm has on other members of the network. In the same manner, we think that the moderation of SRN on the relation of DC and the BA 
and CA of ambidexterity can be both positive and negative.

From the positive side, those firms in the relative central position of the network may strengthen DC's influence on ambidextrous innovation. The reasons are as the following: 1)one of the strengths of the central firms is that they can obtain more resources and diversified knowledge from multiple partners [26]. Those firms that are closer to the center can use less links to access other members of the network, where this distance advantage can help them to access a larger part of heterogeneous resources of the network, and can help them to master, in a more punctual manner, the changes of the industry in question. In this process, firms can continuously improve their ability to sense the environmental changes, and can also help them to better give full play of their resource integration and transformation capabilities, which will eventually promote the ambidextrous innovation. 2) those firms in a more central position can develop new, nonredundant network relations through their influences, and can thus acquire new business opportunities and supplemental resource. In conformity of their rising central position, they are given higher criteria by other members of the network [27], which will help them to fully absorb and reallocate the innovative factors obtained and thus to promote the development of AM. must deal with a larger quantity of information coming from more diversified fields, where the information may come at a greater speed. With the increasing number of these diversified information, SMEs may have difficulty in absorbing and integrating innovative factors obtained, which will certainly reduce the positive influence of DC on ambidexterity. 2) the centrality that surpasses the appropriate level may signify more conflicting views or redundancy [29]. The too central position may receive more conflicting views, which will in turn bring about more pressures that constrains creativity. Furthermore, the central position, too much, will also cause the burden of the increased redundant information and resources, where the innovative factors, if too repetitive, will reduce the marginal benefit of innovation [16].

From these conflicting influences, we suppose that, if a firm's central position in the STN is inferior to the critical value, this will enforce DC's promotion of the BA and CA of ambidexterity; and if its central position in the structural network reaches and surpasses the critical value, the moderation of the STN will be inverted, reducing such correlation. The research hypotheses are as the following.

H3a: STN (Structural Network) plays an inverted $U$ moderation role in the $D C-B A$ relation.

$H 3 b$ : STN plays an inverted U moderation role in the $D C$ - $C A$ relation.

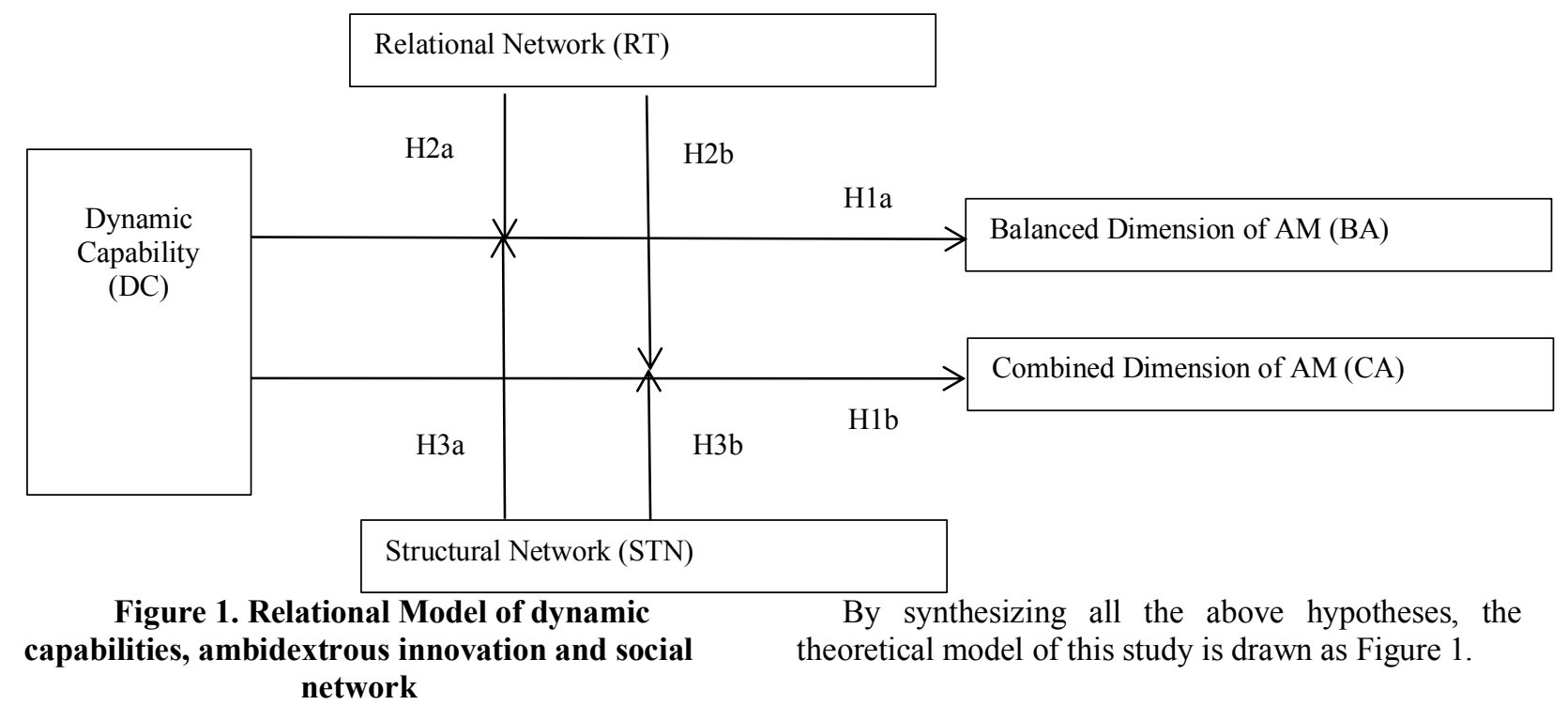

From the negative side, those firms situated too close to the center may reduce DC's influence on AM. The reasons are as the following. 1) from a cognitive point of view, too many relations may reduce a firm's potential to absorb new things [28]. In comparison to those firms not in the central positions, these firms

\section{Research design}

\subsection{Sample and Data}

As this study focuses on the ambidextrous innovation in SMEs, the research has selected its 
sample in line with Rules of SME Classification published in 2011 in mainland China, which is composed of the following 8 industries, where the firms total annual operating revenues (RMB) are between:1) agriculture, 200-0.5 million; 2) industry, 400-3 million; 3) construction and real estate, 800-3 million; 4) wholesale and retailing, 400-1 million; 5) transport and logistics, 300-2 million; 6) hotel and restaurant, 100-1 million; 7) software and IT, 100-0.5 million; and 8) all the other industries where the number of employees is between 300-10.

A questionnaire has been used to collect data. The sample list has been drawn with the help of alumni and students of Lanzhou University's MBA programs. A total of 500 copies have been sent, and 384 have been collected. By a still more careful selection, 36 questionnaires not from SMEs have been eliminated, and the final valid sample consists of 350 questionnaire answers. Before the final survey, 5 SMEs have been chosen to conduct an initial test, where their feedback has been used to realize corrections in the questionnaire, rending the questionnaire more adapted to the context of the current study. The final sample has included 3 regions of China, east, west and south. And $51 \%$ of the correspondents are executives, and $49 \%$ are middle-level managers.

\subsection{Measurement}

The measurement of DC, AM and SN have all been conducted with mature instruments, where a 7-point Likert 7 scale has been utilized. 1-7 signify respectively "total noncompliance" and "full compliance". All the instruments have undergone minor adjustments for readability after the initial test survey.

1) Dynamic Capability (DC). It is accepted to include 3 dimensions of sensing, seizing and reconfiguring opportunities. The measurement of DC in this study has taken into account the contributions of Teece [30]. Each dimension includes finally 5 items, with 15 items in all. 2) Ambidextrous Innovation (AM). The measurement of EPI and ETI have applied the instrument developed by Jansen [9], where, in line with SMEs characteristics, some items have been eliminated. And each of them has 6 items, with 12 items for AM. 3) The Balanced Dimension (BA) and Combined Dimension of CA dimension of AM is evaluated in term of level, where the CA is calculated by the product of the two, in line with the paper of $\mathrm{He}$ \& Wong [31], and the BA is assessed by the formula 1$|x-y| /(x+y)$ by way of the paper by Wang and others [32]. 4) Social network (SN). In accordance with Granovetter's classification of firms' network into two types of relational network (RN) and structural network (STN), we have chosen the following instruments. The $\mathrm{RN}$ is constructed through the studies of Kale [33] with 5 items in all. And the STN is developed by taking the reference of Eisingerich [34], also with 5 items in all. 5) Control variables. In this study, such variables having major impact of SMEs' ambidextrous innovation have been chosen as control variables, which are firm size and firm age. In line with the general practice, the number of employees and the age of creation have been respectively used to evaluate these two control variables.

\subsection{Validity}

Firstly, this study has conducted validity analysis on all the measurements by SPSS25.0. The Cronbach' $\alpha$ coefficients of DC, ERI, ETI, SN and RN are respectively $0.960,0.852,0.854,0.880$, and 0.827 , and the total coefficient is 0.944 , all superior to 0.7 , showing that the measurements have good validity. Secondly, by Lisrel 8.70, a confirmatory factor analysis has been realized, where $\mathrm{KMO}$ value is 0.956 $>0.7$, Sig of Bartlett is 0.000 . The in the test of CFA, five indexes of c2/df, SRMR, CFI, TLI and RMSEA have been assessed, where all the values are within the acceptable range. This has shown the measurements have good polymerization validity and discrimination validity.

\subsection{Common method bias}

When all the items of the questionnaire have been answered by one individual correspondent, due to individual tendency, the correspondent may maintain identical answers to all the similar questions. To test this common method bias, the Harman single factor analysis has been applied in this study. The results show that, in the non-rotated condition, 5 factors have been extracted, and the first factor variance contribution is $37.4 \%<40 \%$. This provides the evidence that this study has no serious common method bias.

\section{Empirical Analysis}

\subsection{Descriptive and correlation analysis}

Table 1 summarizes the mean, standard deviation and correlation coefficients of each factor. As illustrated in Table 1, DC is shown in significant positive correlation with $\mathrm{CA}(\mathrm{r}=0.671, \mathrm{p}<0.01)$ and $\mathrm{BA}$ $(\mathrm{r}=0.128, \mathrm{p}<0.05)$. It can be deduced that our model has relatively good rationality. And the results can undergo further tests. 
Table 1. Descriptive Analysis and correlation coefficients

\begin{tabular}{|l|l|l|l|l|l|l|l|l|l|l|l|}
\hline Variable & Mean & SD & $\mathbf{1}$ & $\mathbf{2}$ & $\mathbf{5}$ & $\mathbf{6}$ & $\mathbf{7}$ & $\mathbf{8}$ & $\mathbf{9}$ & $\mathbf{1 0}$ & $\mathbf{1 1}$ \\
\hline 1 Size & 2.22 & 0.75 & 1 & & & & & & & \\
\hline 2 Age & 2.50 & 0.93 & $0.425^{* *}$ & 1 & & & & & & \\
\hline 3 DC & 4.52 & 1.21 & 0.024 & -0.035 & 1 & & & & & \\
\hline $\begin{array}{l}\text { 4 EPI } \\
\text { Exploratory) }\end{array}$ & 4.08 & 1.34 & 0.048 & -0.010 & $0.717^{* *}$ & 1 & & & & \\
\hline $\begin{array}{l}\text { 5 ETI } \\
\text { (Exploitative) }\end{array}$ & 3.76 & 1.32 & -0.020 & -0.012 & $0.613^{* *}$ & $0.725^{* *}$ & 1 & & & \\
\hline $\begin{array}{l}\text { 6 CA } \\
\text { (Combined) }\end{array}$ & 16.62 & 9.26 & -0.020 & -0.009 & $0.671^{* *}$ & $0.899^{* *}$ & $0.925^{* *}$ & 1 & & \\
\hline $\begin{array}{l}\text { 7 BA } \\
\text { (Balanced) }\end{array}$ & 0.89 & 0.10 & -0.034 & 0.011 & $0.128^{*}$ & 0.089 & $0.373^{* *}$ & $0.304^{* *}$ & 1 & & \\
\hline $\begin{array}{l}\text { 8 RN } \\
\text { (Relational) }\end{array}$ & 3.76 & 1.26 & -0.005 & 0.050 & $0.170^{* *}$ & $0.111^{*}$ & 0.079 & $0.126^{*}$ & 0.008 & 1 & \\
\hline $\begin{array}{l}\text { 9 STN } \\
\text { (Structural) }\end{array}$ & 3.81 & 1.27 & 0.060 & 0.073 & $0.147^{* *}$ & $0.116^{* *}$ & $0.108^{*}$ & $0.148^{* *}$ & 0.056 & $0.681^{* *}$ & 1 \\
\hline
\end{tabular}

Note: ${ }^{*}{ }^{* *}$, and $* * *$ represent respectively $\mathrm{p}<0.05, \mathrm{p}<0.01$, and $\mathrm{p}<0.001$, the following tables are the same.

Table 2. Regression of DC and CA of AM

\begin{tabular}{|c|c|c|c|c|c|c|c|}
\hline & 1a & $2 \mathbf{a}$ & $3 \mathbf{a}$ & $4 a$ & $5 \mathbf{a}$ & 6a & $7 \mathbf{a}$ \\
\hline \multicolumn{8}{|c|}{ 1Control variable } \\
\hline Size & 0.094 & -0.230 & -0.227 & -0.280 & -0.252 & -0.21 & -0.302 \\
\hline Age & -0.051 & 0.271 & 0.268 & 0.156 & 0.237 & 0.234 & 0.172 \\
\hline \multicolumn{8}{|c|}{ 2Independent variable } \\
\hline DC & & $5.075^{* * *}$ & $5.076^{* * *}$ & $8.335^{* * * *}$ & $5.035^{* * *}$ & $8.638^{* * *}$ & $8.847^{* * *}$ \\
\hline \multicolumn{8}{|c|}{ 3Moderation variable } \\
\hline RN & & & 0.015 & 0.530 & & & $0.855^{*}$ \\
\hline STN & & & & & 0.288 & $0.840^{* * *}$ & 0.093 \\
\hline $\mathbf{R N}^{2}$ & & & & $0.401^{*}$ & & & 0.05 \\
\hline STN $^{2}$ & & & & & & $0.389^{*}$ & 0.292 \\
\hline \multicolumn{8}{|c|}{4 Interaction items } \\
\hline $\mathbf{D C} * \mathbf{R N}$ & & & 0.124 & 0.088 & & & 0.133 \\
\hline DC * STN & & & & & 0.162 & 0.027 & 0.042 \\
\hline$D C * \mathbf{R N}^{2}$ & & & & $-.391^{* * *}$ & & & $-0.801^{* *}$ \\
\hline $\mathrm{DC}^{*} \mathbf{S T N}^{2}$ & & & & & & $-.555^{* * *}$ & $-0.840^{* *}$ \\
\hline $\mathbf{F}$ & 0.089 & $48.429^{* *}$ & $41.49^{* * *}$ & $57.633^{* * *}$ & $41.787^{* * *}$ & $60.085^{* * *}$ & $43.653^{* * * *}$ \\
\hline $\mathbf{R}^{2}$ & 0.023 & 0.459 & 0.459 & 0.604 & 0.461 & 0.614 & 0.628 \\
\hline $\mathbf{A R}^{2}$ & 0.012 & 0.451 & 0.448 & 0.594 & 0.450 & 0.604 & 0.614 \\
\hline$\Delta \mathbf{R}^{2}$ & & 0.436 & 0.000 & 0.145 & 0.002 & 0.153 & 0.014 \\
\hline
\end{tabular}

Table 3. Regression results of DC and BA of AM

\begin{tabular}{|c|c|c|c|c|c|c|c|}
\hline & $1 \mathbf{a}$ & $\mathbf{2 a}$ & $3 \mathbf{a}$ & $4 a$ & $6 \mathbf{a}$ & $7 \mathbf{a}$ & $\mathbf{8 a}$ \\
\hline \multicolumn{8}{|c|}{ 1Control variable } \\
\hline Size & -0.006 & -0.007 & -0.007 & -0.006 & -0.007 & -0.008 & -0.007 \\
\hline Age & 0.001 & 0.002 & 0.002 & -0.003 & 0.002 & 0.001 & -0.002 \\
\hline \multicolumn{8}{|c|}{ 2Independent variable } \\
\hline DC & & $0.012^{* *}$ & $0.012^{* *}$ & $0.045^{* * *}$ & $0.011^{*}$ & $0.046^{* * *}$ & $0.050^{* * *}$ \\
\hline \multicolumn{8}{|c|}{ 3Moderation variable } \\
\hline $\mathbf{R N}$ & & & 0.000 & 0.005 & & & -0.001 \\
\hline STN & & & & & 0.005 & $0.009^{*}$ & $0.012^{*}$ \\
\hline $\mathbf{R N}^{2}$ & & & & $-.015^{* * *}$ & & & $-0.011^{* *}$ \\
\hline STN $^{2}$ & & & & & & $-.012^{* * *}$ & -0.006 \\
\hline \multicolumn{8}{|c|}{ 4Interaction item } \\
\hline $\mathbf{D C} * \mathbf{R N}$ & & & 0.000 & 0.004 & & & 0.003 \\
\hline DC * STN & & & & & 0.000 & 0.002 & 0.002 \\
\hline $\mathbf{D C} * \mathbf{R} \mathbf{N}^{2}$ & & & & $-.015^{* * *}$ & & & $-0.010^{* *}$ \\
\hline $\mathrm{DC} * \mathrm{STN}^{2}$ & & & & & & $-.016^{* * *}$ & $-0.008^{*}$ \\
\hline $\mathbf{F}$ & 1.526 & $2.658^{*}$ & 1.888 & $11.450^{* * *}$ & $2.055^{*}$ & $10.972^{* * *}$ & $9.605^{* * *}$ \\
\hline $\mathbf{R}^{2}$ & 0.017 & 0.037 & 0.037 & 0.233 & 0.040 & 0.225 & 0.271 \\
\hline $\mathbf{A R}^{2}$ & 0.006 & 0.023 & 0.018 & 0.212 & 0.021 & 0.205 & 0.243 \\
\hline$\Delta \mathbf{R}^{2}$ & & 0.02 & 0.000 & 0.196 & 0.003 & 0.02 & 0.046 \\
\hline
\end{tabular}




\subsection{Multiple regression analysis}

SPSS25.0 has been utilized to conduct multiple regression analysis to test the relation between DC, $\mathrm{AM}$ and SN. To reduce the issue of high-correlation of interaction item with its constituting variables, the independent and moderation variables have been centralized.

Data shows the positive relation of DC with EPI and ETI, and as this has been in line with the existent researches, this will not be further discussed. The regression results have also confirmed the positive influence of DC on the CA and BA. From the model 2a of Table 2, and model $2 \mathrm{~b}$ from Table 3 , it can be observed that $\mathrm{DC}$ has positive influence both $\mathrm{BA}$ ( $\beta$ $=0.011, \mathrm{P}<0.05)$ and $\mathrm{CA}(\beta=5.140, \mathrm{P}<0.001)$. Hence hypotheses $\mathrm{H1a}$ and $\mathrm{H} 1 \mathrm{~b}$ are supported in our study.

Consequently, in models $4 \mathrm{a}$ and $6 \mathrm{a}$, by way of non-linear moderation, the squared items and their second-order interactions of RN and STN have been analyzed for the inverted $U$ moderation. As shown in model 7a, hypotheses $\mathrm{H} 2 \mathrm{a}$ and $\mathrm{H} 3 \mathrm{a}$ have been supported, where RN ( $\beta=-0.821, \mathrm{p}<0.001)$ and STN ( $\beta=-0.814, p<0.001$ ) have been proven to have an inverted $\mathrm{U}$ moderation on the positive influence of DC on the CA, which is, with an appropriate social network level, the SME's dynamic capabilities have a significant positive impact on the combined dimension of ambidextrous innovation, and while social network level has passed the threshold, the influence of dynamic capabilities on the combined dimension will decrease, and even turn to negative.

The regression results of $\mathrm{DC}, \mathrm{RN}$, and $\mathrm{BA}$ of AM are shown in Table 3. As shown in model 7b, the interaction items of "DC*RN" and "DC*STN" are not significant $(p>0.05)$, non-linear moderation has been analyzed. As the interaction item of squared independent variable and $\mathrm{RN}(\beta=-0.01, \mathrm{p}<0.01)$ and STN $(\beta=-0.008, p<0.05)$ are all significant, $\mathrm{H} 2 \mathrm{~b}$ and $\mathrm{H} 3 \mathrm{~b}$ have been supported, i.e., RN and STN all play an inverted $\mathrm{U}$ moderation on the positive influence of DC on the BA of AM. In other words, with an appropriate social network level, the SME's dynamic capabilities have a significant positive impact on the balanced dimension of ambidextrous innovation, and while social network level has passed the threshold, the influence of dynamic capabilities on the balanced dimension will decrease, and then turn to negative.

To better illustrate the positive moderation of SN on the DC-AM relation, in a more readable manner, this study has drawn slope analysis charts, where the abscissa represents the level of social network, while the ordinate represents the regression coefficients of dynamic capabilities on ambidextrous innovation. Fig
2 is the slope change chart of RN and STN on the CA of DC-AM relation, and Fig 3 is the slope of RN and STN on the BA of DC-AM relation. As shown in Figure 2 and Figure 3, within the critical point range, DC's positive relation with AI is shown in an uptrend, but one surpasses the critical point, this positive relation is then in a down trend, and even have negative influence. At the same time, it can be observed that, the moderation slope of $\mathrm{RN}$ is situated above the STN, which signifies that, under the moderation of RN, DC's positive influence on AM is stronger than that of STN, and that RN has more moderation space than that of STN.

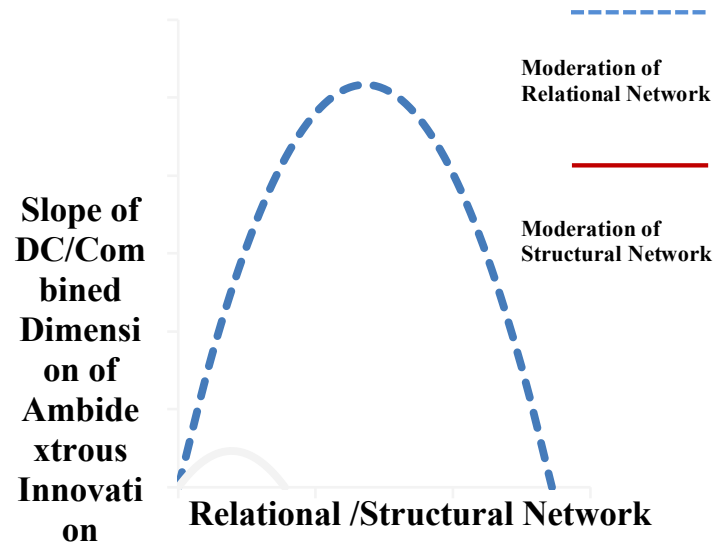

Figure 2. SN's moderation slope of DC-AM (Combined Dimension)

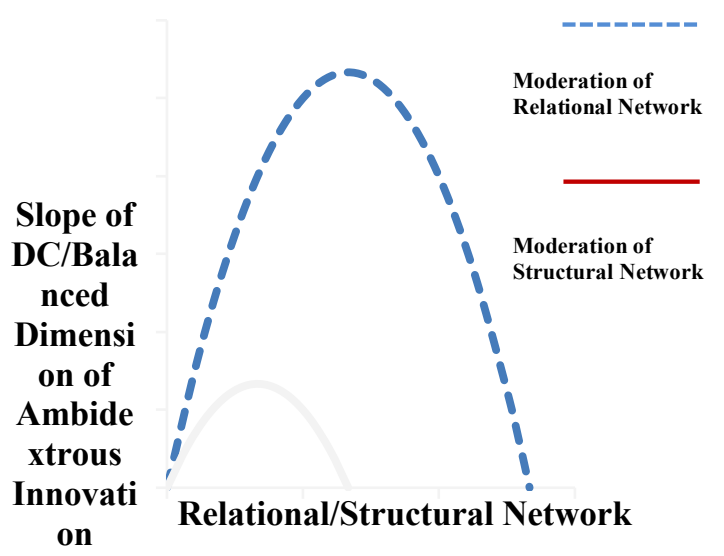

Figure 3. SN's moderation slope of DC-AM (Balanced Dimension)

\section{Discussion}

Taking the SMEs as study object, this study has constructed an effect model of DC and ambidextrous innovation, has discussed the moderation of network on this relation, and has tested the functional 
mechanism of variables through multiple regression. The research results show that:

1) DC is in significant positive relation with both the balanced and combined dimension of ambidextrous innovation;

2) the two dimensions of social network, i.e. relational network and structural network all play an inverted $\mathrm{U}$ moderation on the correlation between DC and ambidexterity, that is to say, when SMEs are embedded, at a certain level, in the relational and structural network, the intensity of these networks may enforce the positive relation between DC and ambidexterity, and once the embeddedness of the relational and structural network has passed the critical value, the increasing intensity of the two network will reduce DC's positive influence on ambidexterity;

3) relational network has a stronger moderation than that of structural network on the DCambidexterity relation, and relational network has a larger moderation space than that of structural network.

\subsection{Theoretical Implications}

1) The study has deepened the DC-ambidexterity researches by offering new evidence of DC's benefits to both the balanced and combined dimension of ambidexterity, and also providing new micro basis for DC's improvements on organizational performance.

Although the existent researches have reached certain consensus on DC's contribution to the establishment of balance mechanism on AM, but subsequent empirical studies on AM has two limitations. Firstly, these studies have deviated from basic ideas of Teece, i.e., DC is the superpower to guide practice and generalize competence [35]. What distinguishes DC from other generic capabilities is that, those firms with DC can flexibly coordinate and regroup those resource/asset and business activities, while reducing, to minimum, the cost of ambidextrous innovation, and balancing, at top management level, ambidexterity and efficiency. However, some scholars has identified DC itself as AM [36], considering ambidexterity as a dimension of DC. This confusion is not helping the construction and deepening of the current theory system. Secondly, the existent studies have been limited themselves on the specific innovation types, which is lack of integrated consideration on the balanced or supplemental mechanism of both EPI and ETI [37].

This study has developed its model in the logic of competence (DC) guiding conduct (AM), has returned to the very essence of Teece's definition of DC, and has thus proven that, DC can guide and promote the flexible adjustments and reconfiguration as well as resources necessary for both EPI and ETI, reaching the balance of and pushing the joint development of the two.

2) The study has integrated DC, SN and AM into one model, where by the two dimensions of SN (RN and STN) as the moderation of the main effect, the analysis and discussion has help to complete the relational model of DC and AM.

The above theoretical and data analysis has demonstrated that, the introduction of $\mathrm{SN}$, as an important external factor, into the relation of DC and AM has significantly improved the effectiveness of the model. Not only the moderation of RN and SRN on the $\mathrm{BA}$ and $\mathrm{CA}$ are significant, these moderations are also proven to be effective in an inverted $U$ shape nonlinear slope. This shows that both the positive and negative conditions of RN and STN should be discussed simultaneously, otherwise, the relative studies will lose its validity.

3) In the DC-AM model, as the moderation of RN is stronger that of STN, this has provided new approaches and evidence for the importance of $\mathrm{RN}$ and STN as external factors.

The slope comparison of Fig. 2 and Fig 3 has shown that, the U slope of STN is more declined, indicating the moderation of STN is not continuous, while $\mathrm{RN}$ has a stronger moderation and is more continued. The authors of this paper have not found other research conclusions and theories to explain this. This is expected to be explored in future studies. The initial explanation of this paper is that, as mainland China is a "guanxi" dominated society, and against this background, RN involves mainly the mutual trust, knowledge and information exchange and intimate individual interaction, which are closely related to firms to acquire new knowledge and expand learning channels. And as the knowledge acquirement and extension of learning channels are helpful to the development of DC [38], they will also be beneficial to innovation. In this way, firms can harvest gains, punctually, from the establishment of RN. Similarly, the damage to DC and the constrains of innovation caused by too complicated network and too much redundant information will also be remarkable.

4) This study has provided evidence that DC can help SMEs to overcome the path dependence on ETI, where they can also realize the balance and complementation of EPI and ETI by engaging in EPI. This has further supported the application of DC theories in the context of SMEs' innovation management.

\subsection{Managerial Implications}

The research conclusions of this study have some insights on SMEs' ambidextrous innovation. 
DC theories can be used by SMEs to balance and coordinate their internal ambidexterity. As DC is regarded as high-rank capability, which is superior to common managerial abilities, it can guide and control their behavior to upgrade their capability to confront environmental uncertainty. It can also be treated as a dynamic process to sense, seize and reconfigure opportunities as well as develop unique resources. If the mangers in SMEs can effectively utilize DC, they can be more sensitive to market opportunities, quicker to integrate external knowledge and to provide technical solutions, which will promote the exploratory innovation. And the business process formed in the exploratory innovation can be configured and upgraded to those routine procedures and internal knowledge system of exploitative innovation. Meanwhile, DC can also improve the quality of exploitative innovation, which will help to support exploratory innovation with procedures and protocols developed through exploitative innovation. And this will eventually help the balance and combination of exploratory and exploitative innovation.

SMEs should maintain an appropriate position in the social network. Their strategies should be avoiding the marginalization, and as the center as well. On one hand, they should get closer to the center of social network to develop high-level cooperation and to acquire best practice, new and innovative ideas necessary for exploratory innovation; and on the other hand, they should keep clear of high structural embeddedness. They should systematically monitor the changes of their positional advantage and punctually upgrade the configuration information. These behaviors can help them to be aware of competence and position changes of other members of the network, and this will give them clearer idea of their position in the network, and help them to better react to threats and uncertainties.

SMEs should maintain an appropriate level of connections with the members of the social network. For one thing, they should actively introduce new partners, and reinforce their cooperation with informal relation with other firms, universities, and research centers, the keeping of sound relation with them will help SMEs to enter larger and wider markets, to acquire sufficient key resources, and to promote the exploratory innovation. For the other, too close SN will consume SMEs' time and energy to deal with redundancy, as with only limited competence, it will become difficult for them integrate these innovative factors into their internal system, which will then hinder innovation. As a result, SMEs should keep suitable relation with other members of the social network, but not with too much time and energy.
It is noteworthy that the impact of changes in SMEs' relational network on the DC-Ambidexterity relation will be more remarkable. In business practice, there will arise a dilemma of "guanxi", the effective utilization of RN will promote DC's coordination on ambidextrous innovation, while too complicated relational network will destroy or reduce this positive influence.

\subsection{Further research and Limitations}

As this study has collected only cross-section data, the causal relation between DC and ambidexterity will be limited. A further vertical study may provide more insights on the basic model. Future studies can be organized in a time-line, where mechanism of influence can be investigated for different periods. Besides, as this research has found remarkable differences of moderation in relational network and structural network, and has explained this difference in the context of mainland China, will this difference be observed in different countries, cultures, and contexts remain an interesting domain to explore more.

\section{References}

[1] E. Danneels, "The dynamics of product innovation and firm competences", Strategic Management Journal, 2002, 23(12), pp.1095-1121.

[2] D. J. Teece, G. Pisano, and A. Shuen, "Dynamic capabilities and strategic management", Strategic Management Journal, 1997, 18(7), pp. 509-533.

[3] M. L. Sheng, "A dynamic capabilities-based framework of organizational sensemaking through combinative capabilities towards exploratory and exploitative product innovation in turbulent environments", Industrial Marketing Management, 2017, 65, pp.28-38.

[4] H. W. Ho, P. N. Ghauri, and J. A. Larimo, "Institutional distance and knowledge acquisition in international buyersupplier relationships: The moderating role of trust", Asia Pacific Journal of Management, 2018.

[5] S. Wang, R. Guidice, Y. Zhou et al., "It's more complicated than we think: The implications of social capital on innovation", Asia Pacific Journal of Management, 2016.

[6] C. R. Li , C. J. Lin, H. C. Huang , "Top management team social capital, exploration-based innovation, and exploitation-based innovation in SMEs", Technology Analysis \& Strategic Management, 2014, 26(1), pp.69-85.

[7] C. M. Mcdermott and D. I. Prajogo, "Service innovation and performance in SMEs", International Journal of Operations \& Production Management, 2012, 32(2), pp.216237.

[8] O. Koryak, A. Lockett, J. Hayton et al., "Disentangling the antecedents of ambidexterity: Exploration and exploitation”, Research Policy, 2018, 47(2), pp.413-427.

[9] J. P. Jansen, V. Dbfaj, and H. W. Volberda, "Exploratory Innovation, Exploitative Innovation, and Performance: 
Effects of Organizational Antecedents and Environmental Moderators", Firm Report, 2006, 52(11), pp.1661-1674.

[10] Q. Cao, E. Gedajlovic, and H. Zhang, "Unpacking Organizational Ambidexterity: Dimensions, Contingencies, and Synergistic Effects", Organization Science, 2009, 20(4), pp.781-796.

[11] N. Kammerlander, D. Burger, A. Fust et al., "Exploration and exploitation in established small and medium-sized enterprises: The effect of CEOs' regulatory focus", Journal of Business Venturing, 2015, 30(4), pp.582602.

[12] D. J. Teece, "Towards a capability theory of (innovating) firms: implications for management and policy, Cambridge Journal of Economics, 2017, 41(3):693-720.

[13] L. Jurksiene and A. Pundziene, "The relationship between dynamic capabilities and firm competitive advantage The mediating role of organizational ambidexterity”, European Business Review, 2016, 28(4), pp.431-448.

[14] M. Asif, P. Murphy, and K. Bruce, "Exploring the Antecedents of Ambidexterity: A Taxonomic Approach", Management Decision, 2017.

[15] J. Carrick, "R\&D resources development in life sciences ventures: a dynamic capabilities perspective", Journal of Small Business and Enterprise Development, 2016, 23(4), pp.1191-1207.

[16] B. Mcevily, G. Soda, and M. Tortoriello, "More Formally: Rediscovering the Missing Link between Formal Organization and Informal Social Structure", The Academy of Management Annals, 2014, 8(1), pp.299-345.

[17] A. Ioanida, D. Corina, et al., "The impact of social networks on SMEs' innovation potential", Procedia Manufacturing, 2018, 22(2), pp. 936-941

[18] M.S. Granovetter, "Problems of explanation in economic sociology", In N. Nohria \& R. Eccles (Eds.), Networks and organizations: Structure, form, and action, Boston, MA: Harvard Business School Press, 1992, pp.2526). .

[19] G. Villaverde, P. M. Rodrigo, J. Requena, and M. J. Ortega, "Technological dynamism and entrepreneurial orientation: the heterogeneous effects of social capital", Journal of Business Research, 2018, 83, pp.51-64.

[20] S. Fainshmidt, M.L. Frazier, "What Facilitates Dynamic Capabilities? The Role of Organizational Climate for Trust", Long Range Planning, 2016:S0024630116300371.

[21] M. T. Rivera, S. B. Soderstrom, and B. Uzzi, "Dynamics of Dyads in Social Networks: Assortative, Relational, and Proximity Mechanisms", Annual Review of Sociology, 2010, 36(1), pp. 91-115.

[22] M.S.Granovetter, "The Strength of Weak Ties[J]. American Journal of Sociology”, 1973, 78(6), 1360-1380.

[23] M. Eitan and P. Renana, "The effect of social networks structure on innovation performance: A review and directions for research", International Journal of Research in Marketing, 2018, 36(1), pp.3-19.
[24] H.V. Villena, E. Revilla, T.Y. Choi, "The dark side of buyer-supplier relationships: A social capital perspective", Journal of Operations Management, 2011, 29(6), pp.561-576. [25] R. Gulati, "Alliances and networks", Strategic Management Journal, 1998, 19(4), p.25.

[26] J. Zang J. Structural holes, exploratory innovation and exploitative innovation[J]. Management Decision, 2018(2).

[27] J.E. Perry-Smith and C. E. Shalley, "The social side of creativity: a static and dynamic social network perspective", Academy of Management Review, 2003, 28(1), pp.89-106.

[28] V. Gilsing, B. Nooteboom, W. Vanhaverbeke, "Network embeddedness and the exploration of novel technologies : technological distance, betweenness centrality and density", Research Policy, 2008, 37(10), pp.1717-1731.

[29] M.J. Ruiz-Ortega, P.M. Villaverde, G. Parra-Requena, "How structural embeddedness leads to pioneering orientation", Technological Forecasting \& Social Change, 2018.

[30] A. Shuen , P.F. Feiler, and D.J. Teece, "Dynamic capabilities in the upstream oil and gas sector: Managing next generation competition", Energy Strategy Reviews, 2014, 3, pp.5-13.

[31] Z. L. He and P. K. Wong, "Exploration vs. Exploitation: An Empirical Test of the Ambidexterity Hypothesis", Organization Science, 2004, 15(4), pp.481-494.

[32] F. B. Wang, J. X. Chen, and Y. Yang, "An Analysis of the Effect of the Explorative and Exploitative Innovations and their Balance", Management World (in Chinese), 2013, 3, pp. 96-112.

[33] P. Kale, H. Singh, and H. Perlmutter, "Learning and protection of proprietary assets in strategic alliances: building relational capital", Strategic Management Journal, 2000, 21(3), pp.217-237.

[34] A. Eisingerich, S. Bell, P. Tracey, "How can clusters sustain performance? The role of network strength, network openness, and environmental uncertainty", Research Policy, 2010, 39(2), 239-253.

[35] D. J. Teece and S. Leih, "Uncertainty, innovation, and dynamic capabilities: an introduction", California Management Review, 2016, 58(4), pp.5-12.

[36] A. Kriz, R. Voola, and U. Yuksel, "The dynamic capability of ambidexterity in hypercompetition: qualitative insights", Journal of Strategic Marketing, 2014, 22(4), pp.287-299.

[37] S. Raisch, J. Birkinshaw, G. Probst, et al., "Organizational Ambidexterity: Balancing Exploitation and Exploration for Sustained Performance", Organization Science, 2009, 20(4), pp.685-695.

[38] X. He, S.Z. Huang, K.Zhao, et al., "The Relationship between Learning Orientation and Dynamic Capability based on Environmental Education", Eurasia Journal of Mathematics, Science and Technology Education, 2018, 14(6), pp.2193-2202. 